\title{
Analysis of intellectual and cognitive performance in patients with multi-infarct dementia, vertebrobasilar insufficiency with dementia, and Alzheimer's disease ${ }^{1}$
}

\author{
F. I. PEREZ, ${ }^{2}$ V. M. RIVERA, J. S. MEYER, J. R. A. GAY, \\ R. L. TAYLOR, AND N. T. MATHEW \\ From the Departments of Neurology and Psychiatry, Baylor College of Medicine, and the \\ Baylor-Methodist Center for Cerebrovascular Research, Houston, Texas, U.S.A.
}

SYNOPSIS A prominent feature in dementia is intellectual deterioration. Review of the clinical literature indicates a lack of suitably quantitated studies of specific intellectual defects in dementia. The present study investigated the performance of patients with multi-infarct dementia (MID), dementia due to Alzheimer's disease (AD), and vertebrobasilar insufficiency (VBI) with dementia using the Wechsler Adult Intelligence Scale (WAIS). Forty-two patients ranging in age from 45 to 85 years $(\bar{x} 66)$ were included. Significant differences in cognitive and intellectual performance were found between patients with dementia due to VBI and MID versus neuronal atrophy of the Alzheimer's type. The group with AD performed significantly and consistently lower on all measures. There were no significant differences between the two cerebrovascular disease groups, even though the MID group performed consistently more poorly than the VBI group. A discriminant function analysis classified $74 \%$ of the patients correctly based on the individual WAIS scores. The diagnosis was more easily made when tasks measuring visual motor coordination and abstract reasoning were included in the analysis.

Dementia is a syndrome of behavioural and cognitive reduction caused by diffuse cerebral dysfunction. C. M. Fisher (1968) defines dementia as a chronic reduction in any or all of the higher verbal and non-verbal cerebral functions. According to Hachinski et al. (1974), the first step in the investigation of a demented patient is to document the nature and extent of the organic intellectual deterioration. We believe this is best achieved by a battery of neuropsychological tests which quantitate the deficit in higher cortical functions.

Most authors agree that a prominent feature in dementia is intellectual deterioration or reduction in the intelligence quotient (IQ). However, review of the clinical literature indicates a lack of

\footnotetext{
1 This work was supported by Grant NS 09287 from the National Institute of Neurological Diseases and Stroke, and in part from NIH grant RR-00259 for computational activities.

2 Reprint requests to: Francisco I. Perez, PhD, Department of Neurology, Baylor College of Medicine, 1200 Moursund Avenue, Houston, Texas, 77025, U.S.A.

(Accepted 16 January 1975.)
}

suitably quantitated and objective investigative studies dealing with identification, analysis and individual quantification of specific intellectual defects in dementia. Karp (1974) defines dementia as a 'deterioration or loss of intellectual function' but this is not a full description of the syndrome. Intelligence is not a unitary function but is rather a matrix of interrelated capacities of the entire cerebrum. Most intellectual measures of neuropsychological testing include functions such as problem-solving, verbal capacity, numerical reasoning, abstract thinking, learning new material, as well as visual-spatial planning ability, and visual-motor coordination.

Recently, progress has been made in delineating characteristic pathological, biochemical, as well as regional cerebral blood flow and metabolic differences and patterns in the various diseases producing the syndrome of dementia. These studies have especially advanced the diagnostic identification and management of demen- 
tias due to cerebrovascular disease (multi-infarct dementia), normal pressure hydrocephalus, and dementias due to neuronal atrophies (Rivera and Meyer, in press). Not much progress has been made, however, in the precise quantification of intellectual performance and the natural history of its course by serial neuropsychological examinations in the dementias. It is our hypothesis that different diseases produce different patterns of dementia and hence produce different quantitative scores on currently designed multiple tests of intellectual and cognitive measures.

It is generally accepted that both 'presenile' and 'senile' dementia are forms of Alzheimer's disease with the same clinical manifestations and histopathological findings (neuronal atrophy, neurofibrillary tangles, plaques, etc.). This form of dementia is definitely not due to or associated with arteriosclerosis (Hachinski et al., 1974).

In hypertensive patients or those associated with other risk factors for cerebrovascular disease such as diabetes mellitus, hyperlipidaemia, and disseminated intravascular coagulation, multiple episodes of cerebral ischaemia and infarctions may occur with cumulative and hence progressive neurological deficits with subsequent mental deterioration. This is a wellrecognized form of dementia that was termed multi-infarct dementia by Hachinski et al. (1974).

Vertebrobasilar arterial insufficiency may also be a cause of progressive memory loss and dementia due to multiple attacks of ischaemia and infarctions in the para-amygdaloid areas and other limbic and cortical structures irrigated by the posterior cerebral circulation (Rivera et al., 1974). A significant percentage of these patients in their clinical course have attacks of transient global amnesia and eventually may suffer an 'amnesic stroke' with permanent loss of memory, sometimes associated with visual field defects due to occlusion of one or both posterior cerebral arteries (Mathew and Meyer, 1974).

The purpose of the present study is to investigate the performance of these three groups of patients with different types and aetiologies of dementia using the various subtests of the Wechsler Adult Intelligence Scale (WAIS). The WAIS is the most widely used of the individually administered tests designed to study adult intelligence. A detailed discussion of its clinical usefulness in evaluating the disability caused by the organic dementias is well summarized by Horenstein (1971).

\section{METHOD}

PATIENT SELECTION Forty-two patients, 17 men and 25 women ranging in age from 45 to 85 with a mean age of 66 years, were admitted to the study. All were patients admitted to the Baylor-Methodist Center for Cerebrovascular Research, and the majority were followed up for at least a year or more with numerous re-examinations.

One of the authors (Dr V. Rivera, a neurologist) first classified the patients into three diagnostic groups on the basis of personal evaluation and review of the entire diagnostic evaluation. This required careful inspection of the records of over 100 demented patients, and the 42 patients were admitted to the study after the diagnosis was objectively confirmed. Only patients with an uncomplicated diagnosis of vertebrobasilar insufficiency (VBI), rather than a mixed diagnosis of VBI and some other cerebral disorder-for example, completed stroke or carotid disease-were included. In two cases the diagnosis of Alzheimer's disease (AD) was confirmed by necropsy.

CLINICAL DIAGNOSTIC ClASSIFICATION The following were the criteria used for establishing the diagnosis and classifying the patients into three groups of dementias. In all cases, systemic disorders causing dementia were excluded by such appropriate tests as those for neurosyphilis, vitamin B deficiency, folate deficiency, uraemia, and hypothyroidism.

ALZHEIMER'S DISEASE (AD) These patients presented a history of chronic progressive dementing process without risk factors (Kannel et al., 1971) or evidence of cerebrovascular disease. The clinical course in these patients was not characterized by episodic worsening of mentation as typically occurs in patients with cerebrovascular disease. There was no history of transient cerebral ischaemic attacks, but a steadily progressive deterioration of intellectual functions.

The general medical examination gave no evidence of arteriosclerosis elsewhere in the body such as arteriosclerotic heart disease or ischaemia of the extremities. The neurological examination gave evidence primarily of frontal lobe and corticobulbar signs and evidence of parietal lobe disorders such as dyspraxia, dysphasia, visuospatial discrimination, and dyslexia. The signs of cerebral dysfunction were diffuse and bilateral. Grasp, sucking, and glabellar reflexes were usually prominent.

Cisternography and regional cerebral blood flow 
TABLE 1

ABILITIES MEASURED BY VERBAL AND NON-VERBAL SUBTESTS OF WAIS*

\begin{tabular}{ll}
\hline & \multicolumn{1}{c}{ Abilities } \\
\hline $\begin{array}{l}\text { Verbal subtest } \\
\text { 1. Information }\end{array}$ & $\begin{array}{l}\text { 1. Fund or knowledge of general cultural } \\
\text { information; remote memory }\end{array}$ \\
2. Arithmetic & $\begin{array}{l}\text { 2. Arithmetical or numerical reasoning } \\
\text { ability; concentration }\end{array}$ \\
3. Similarities & $\begin{array}{l}\text { 3. Abstract verbal reasoning; concept } \\
\text { formation }\end{array}$ \\
$\begin{array}{c}\text { Non-verbal subtest } \\
\text { 1. Picture completion }\end{array}$ & $\begin{array}{l}\text { 1. Ability to differentiate essential from } \\
\text { non-essential details }\end{array}$ \\
2. Block design & $\begin{array}{l}\text { 2. Visual motor coordination; visual } \\
\text { organization }\end{array}$ \\
3. Picture arrangement & \begin{tabular}{l} 
3. Visual spatial planning ability \\
\hline
\end{tabular}
\end{tabular}

* Wechsler (1958).

studies before and after CSF removal (Mathew et al., 1975) were performed with Ytterbium ${ }^{169}$ and excluded the diagnosis of normal pressure hydrocephalus. Aortocranial angiograms ruled out cerebrovascular disease, chronic subdural haematoma, or cerebral neoplasm. Pneumoencephalograms showed cortical atrophy predominantly in the frontal and parietal regions, and the thalamostriate vein configuration showed enlarged ventricles.

MULTI-INFARCT DEMENTIA (MID) The dementing process in these patients was associated with documented risk factors for cerebrovascular disease (Kannel et al., 1971), particularly a long-standing history of hypertension. The clinical course of the dementia was characterized by episodic strokes with cumulative worsening of mentation plus associated transient cerebral ischaemic episodes in either the carotid or vertebrobasilar arterial territories or both.

In the history and/or documented by the general medical examination there was evidence of arteriosclerotic disease elsewhere in the body in every case. These included: angina, myocardial infarction, intermittent claudication, arterial bruits, abnormal electrocardiograms, etc. The neurological examination also showed multiple signs of diffusely represented cerebral deficits attributable to multiple vascular lesions such as hemiplegia, hemiparesis, alternating hemiparesis, dysphasia, hemianopia, or cortical sensory loss confined to segmental zones of half the body.

Aortocranial arteriography confirmed the diagnosis by demonstrating arteriosclerotic plaques and/ or occluded cerebral vessels.

VERTEBROBASILAR ARTERIAL INSUFFICIENCY WITH DEMENTIA (VBI) The same criteria as for MID were present, but the clinical manifestations clearly arose from multiple episodes of infarcts and ischaemia in the distribution of supply of the vertebrobasilar system. Typically, these patients suffered from: vertigo, dizziness, intermittent ataxia, drop attacks, episodes of transient global amnesia, nystagmus, hemianopia, photopsia, etc., plus a step-wise deterioration of memory and higher cortical functions.

Aortocranial arteriograms showed prominent lesions in the posterior circulation, such as stenosis of the cerebral arteries, plaques in the basilar artery and disease of the posterior cerebral arteries.

NEUROPSYCHOLOGICAL TEST PROCEDURE The data utilized in the present study were derived from the short form of the WAIS developed at Baylor College of Medicine, specifically modified for the examination of elderly patients (Baer and Gaitz, 1971). This form comprised six of the 11 WAIS subtests. These included the following three verbal subtests: Information, Arithmetic, and Similarities. Three nonverbal subtests were used-Picture Completion, Block Design, and Picture Arrangement. Table 1 summarizes those aspects of higher cortical function measured by these subtests (Wechsler, 1958). A Full Scale IQ, Performance IQ, Verbal IQ, and raw scores for each of the individual subtests were obtained on every patient.

\section{RESULTS}

ANALYSIS OF COVARIANCE AND SCHEFFÉ TEST An analysis of covariance was performed in order to control such factors as possible effects of age and education on the performance tested between the three diagnostic categories (Table 2). The AD group tended to be younger than the other two groups. The mean education for the VBI and the MID groups was not different, but the AD group showed a significantly higher level of education,

TABLE 2

MEANS AND STANDARD DEVIATIONS OF AGE AND EDUCATION

\begin{tabular}{|c|c|c|c|c|}
\hline & \multicolumn{2}{|c|}{$\operatorname{Age}(y r)$} & \multicolumn{2}{|c|}{ Education } \\
\hline & Mean & $S D$ & Mean & $S D$ \\
\hline Multi-infarct & 69.25 & 11.428 & 10.250 & 4.024 \\
\hline Alzheimer's disease & 62.20 & 11.429 & 13.700 & 3.529 \\
\hline VBI & 65.31 & 8.404 & 10.062 & 4.040 \\
\hline
\end{tabular}

$\mathrm{SD}=$ Standard deviation 
TABLE 3

RESULTS OF ANALYSIS OF COVARIANCE

\begin{tabular}{|c|c|c|c|c|c|c|c|}
\hline \multirow[t]{2}{*}{ Variables } & \multicolumn{2}{|c|}{ Multi-infarct } & \multicolumn{2}{|c|}{ Alzheimer's } & \multicolumn{2}{|c|}{$V B I$} & \multirow[t]{2}{*}{ Fstatistic } \\
\hline & Mean* & $S E^{*}$ & Mean* & $S E^{*}$ & Mean* & $S E^{*}$ & \\
\hline $\begin{array}{l}\text { 1. Verbal IQ } \\
\text { 2. Performance } \\
\text { 3. Full Scale IQ } \\
\text { 4. Information } \\
\text { 5. Arithmetic } \\
\text { 6. Similarities } \\
\text { 7. Picture completion } \\
\text { 8. Block design } \\
\text { 9. Picture arrangement }\end{array}$ & $\begin{array}{r}89.22 \\
86.70 \\
87.68 \\
11.58 \\
6.94 \\
7.24 \\
5.86 \\
14.27 \\
9.48\end{array}$ & $\begin{array}{l}4.43 \\
3.53 \\
3.96 \\
1.34 \\
0.87 \\
1.35 \\
0.87 \\
2.00 \\
1.25\end{array}$ & $\begin{array}{r}65.86 \\
66.99 \\
64.58 \\
4.87 \\
2.80 \\
1.44 \\
2.18 \\
3.22 \\
3.27\end{array}$ & $\begin{array}{l}5.83 \\
4.64 \\
5.21 \\
1.77 \\
1.15 \\
1.78 \\
1.15 \\
2.63 \\
1.64\end{array}$ & $\begin{array}{r}97.49 \\
96.87 \\
97.15 \\
14.13 \\
8.87 \\
9.04 \\
8.40 \\
20.90 \\
11.72\end{array}$ & $\begin{array}{l}4.40 \\
3.50 \\
3.93 \\
1.34 \\
0.87 \\
1.34 \\
0.87 \\
1.99 \\
1.24\end{array}$ & $\begin{array}{r}9.122 \dagger \\
12.686 \dagger \\
12.018 \dagger \\
8.471 \dagger \\
8.572 \dagger \\
5.725 \ddagger \\
9.042 \dagger \\
13.83 \dagger \\
8.170 \dagger \\
\end{array}$ \\
\hline
\end{tabular}

having on the average three years additional education. Table 3 shows the results of the analysis of covariance for each of the WAIS subtests. Significant differences were found among the groups for each of the variables.

In order to determine any specific differences among the groups for each of the variables examined, a Scheffé test for ad hoc comparisons was performed. The results are shown in Table 4. No significant differences were found between the MID and VBI groups on any of the variables tested. It was of particular significance and clinical importance that the $\mathrm{AD}$ and the VBI groups were significantly different for all the subtests included $(P<0.01)$. Likewise, the MID and the $\mathrm{AD}$ groups showed significant difference $(\mathrm{P}<0.01-\mathrm{P}<0.05)$ for all the variables with the

TABLE 4

RESULTS OF SCHEFFÉ TEST TO DETERMINE SIGNIFICANCE BETWEEN SPECIFIC GROUPS

\begin{tabular}{|c|c|c|c|}
\hline Variables & $\begin{array}{c}\text { Multi-infarcts } \\
\text { vs. } \\
\text { Alzheimer's }\end{array}$ & $\begin{array}{l}\text { Multi-infarct } \\
\text { vs. VBI }\end{array}$ & $\begin{array}{c}\text { Alzheimer's } \\
\text { vs. VBI }\end{array}$ \\
\hline $\begin{array}{l}\text { 1. Verbal IQ } \\
\text { 2. Performance IQ } \\
\text { 3. Full Scale IQ } \\
\text { 4. Information } \\
\text { 5. Arithmetic } \\
\text { 6. Similarities } \\
\text { 7. Picture completion } \\
\text { 8. Block design } \\
\text { 9. Picture arrangement }\end{array}$ & $\begin{array}{l}P<0.05 \\
P<0.01 \\
P<0.01 \\
P<0.05 \\
P<0.05 \\
P<0.05 \\
P>0.05 \\
P<0.01 \\
P<0.05\end{array}$ & $\begin{array}{l}P>0.05^{*} \\
P>0.05^{*} \\
P>0.05^{*} \\
P>0.05^{*} \\
P>0.05^{*} \\
P>0.05^{*} \\
P>0.05^{*} \\
P>0.05^{*} \\
P>0.05^{*}\end{array}$ & $\begin{array}{l}P<0.01 \\
P<0.01 \\
P<0.01 \\
P<0.01 \\
P<0.01 \\
P<0.01 \\
P<0.01 \\
P<0.01 \\
P<0.01\end{array}$ \\
\hline
\end{tabular}

* Not significant.
TABLE 5

PREDICTIVE CLASSIFICATION INTO DIAGNOSTIC GROUPS

\begin{tabular}{ccccc}
\hline Positives & Multi-infarct & Alzheimer's & VBI & Total \\
\hline Valid & $9(56 \%)$ & $9(90 \%)$ & $13(81 \%)$ & $31(74 \%)$ \\
False & $7(44 \%)$ & $1(10 \%)$ & $3(19 \%)$ & $11(26 \%)$ \\
\hline
\end{tabular}

exception of the Picture Completion subtest where there was no significant difference.

DISCRIMINANT FUNCTION ANALYSIS A discriminant function analysis (R. A. Fisher, 1936) was performed on 11 predictor variables (see Table 6) and the three diagnostic groups. The multiple discriminant analysis produced a set of coefficients or weights for the various dependent measures which best separate or discriminate the three different diagnostic categories. The between group variance is maximized relative to the within group variance. A composite discriminant predictor score was then computed for each patient based on his or her raw scores and the optimal lambda weightings $(\lambda)$ for each variable. Based on this composite score, each patient was then classified into one of the three diagnostic categories.

A Mahalanobis generalized distance test (delta squared- $D^{2}$ ) was then performed in order to measure the degree of separation of the probability distributions of the three diagnostic groups. The Mahalanobis $\mathrm{D}^{2}$ was significant $\left(\mathrm{D}^{2}=78.66\right.$, 
$\mathrm{d} \cdot \mathrm{f}=22, \quad \mathrm{P}<0.001$ ), and thus permitted the assignment of patients to the different diagnostic categories. The probability distributions of the three diagnostic groups were found to be discrete for each diagnostic category. Table 5 presents the assignment of patients to each diagnostic group based on the 11 predictor variables. Examination of Table 5 reveals that 31 patients $(N=42)$ were correctly diagnosed as VBI, MID, or AD by the WAIS subtests alone, yielding a valid positive hit rate of $74 \%$ and a false positive rate of $26 \%$.

STEP-WISE REGRESSION ANALYSIS The purpose of this analysis was to determine the ranking of the predictor variables in terms of their ability to discriminate among the three diagnostic groups. The variable ranking, $F$ statistic for each variable and cumulative $F$ statistic (the F statistic for the given variable and all the variables above it) are presented in Table 6. Block Design revealed the highest discriminant ranking. Similarities, Performance IQ, Information, and Picture Arrangement ranked second, third, fourth, and fifth respectively. The combination of Block Design and Similarities increased the diagnostic accuracy (hit rate). The inclusion of Performance IQ, Information, and Picture Arrangement decreased the hit rate slightly. The remaining six variables contributed minimally.

\section{DISCUSSION}

The present results indicate that there are significant differences in cognitive and intellectual performance between patients with dementia due to VBI and MID versus neuronal atrophy of the Alzheimer's type. The Figure shows the agecorrected scaled scores of the various WAIS subtests for the three groups. A scaled score of 10 indicates average performance. The Figure shows that the group with AD performed significantly and consistently lower on all measures even though they tended to be younger and to have significantly more years of formal education (Table 2). There were no significant differences between the two cerebrovascular disease groups, even though the MID group performed consistently more poorly than the VBI group. No particular pattern of cognitive deficit was indicated. The present findings suggest that the
TABLE 6

STEP-WISE REGRESSION OF DISCRIMINANT FUNCTION ANALYSIS

\begin{tabular}{lclll}
\hline \multicolumn{1}{c}{ Ranked variables } & F statistic of variable & Cumulative F statistic \\
\hline 1. Block design & $\mathrm{F}(1,40)$ & $5.901^{*}$ & $\mathrm{~F}(1,40)$ & $5.901^{*}$ \\
2. Similarities & $\mathrm{F}(1,39)$ & $4.801^{*}$ & $\mathrm{~F}(2,39)$ & $5.631^{*}$ \\
3. Performance IQ & $\mathrm{F}(1,38)$ & 0.947 & $\mathrm{~F}(3,38)$ & $4.065^{*}$ \\
4. Information & $\mathrm{F}(1,37)$ & 0.292 & $\mathrm{~F}(4,37)$ & $3.065^{*}$ \\
5. Picture arrangement & $\mathrm{F}(1,36)$ & 0.452 & $\mathrm{~F}(5,36)$ & $2.506^{*}$ \\
6. Verbal IQ & $\mathrm{F}(1,35)$ & 0.252 & $\mathrm{~F}(6,35)$ & 2.087 \\
7. Full IQ & $\mathrm{F}(1,34)$ & 0.222 & $\mathrm{~F}(7,34)$ & 1.781 \\
8. Age & $\mathrm{F}(1,33)$ & 0.267 & $\mathrm{~F}(8,33)$ & 1.558 \\
9. Education & $\mathrm{F}(1,32)$ & 0.232 & $\mathrm{~F}(9,32)$ & 1.378 \\
10. Picture completion & $\mathrm{F}(1,31)$ & 0.187 & $\mathrm{~F}(10,31)$ & 1.228 \\
11. Arithmetic & $\mathrm{F}(1,30)$ & 0.197 & & \\
\hline
\end{tabular}

$* \mathbf{P}<0.05$.

cognitive and intellectual deficits are similar for the three disorders but with differences in the degree of impairment.

The results of the discriminant function analysis performed on 11 predictor variables (Table 6) and on the three diagnostic groups are encouraging. Thirty-one $(74 \%)$ out of 42 patients were correctly diagnosed as VBI, MID, or AD purely on the basis of these intellectual and cognitive variables. It is important to note that $90 \%$ (nine out of 10 ) of the AD patients and $81 \%(13$ out of 16) of the VBI patients were correctly classified, while only $56 \%$ (nine out of 16 ) of the MID group were correctly diagnosed. These results indicate that it is easier to classify patients who are either maximally disordered (AD) or minimally disordered (VBI) from a cognitive viewpoint. The data suggest that the MID group is less homogeneous than the other two groups, as might be predicted from the patchy nature of the disease process. The degree and pattern of cognitive deficit varies with each MID patient depending on the sites, location, extent, and number of cerebral infarctions. Of the seven MID patients who were misclassified, four were classified as VBI and three as AD. The one misclassified patient in the AD group was incorrectly identified as MID. All patients misclassified in the VBI group (three) were identified as MID.

The step-wise regression analysis was performed in order to determine the ranking of the predictor variables in terms of their ability to discriminate among the three clinical diagnostic 


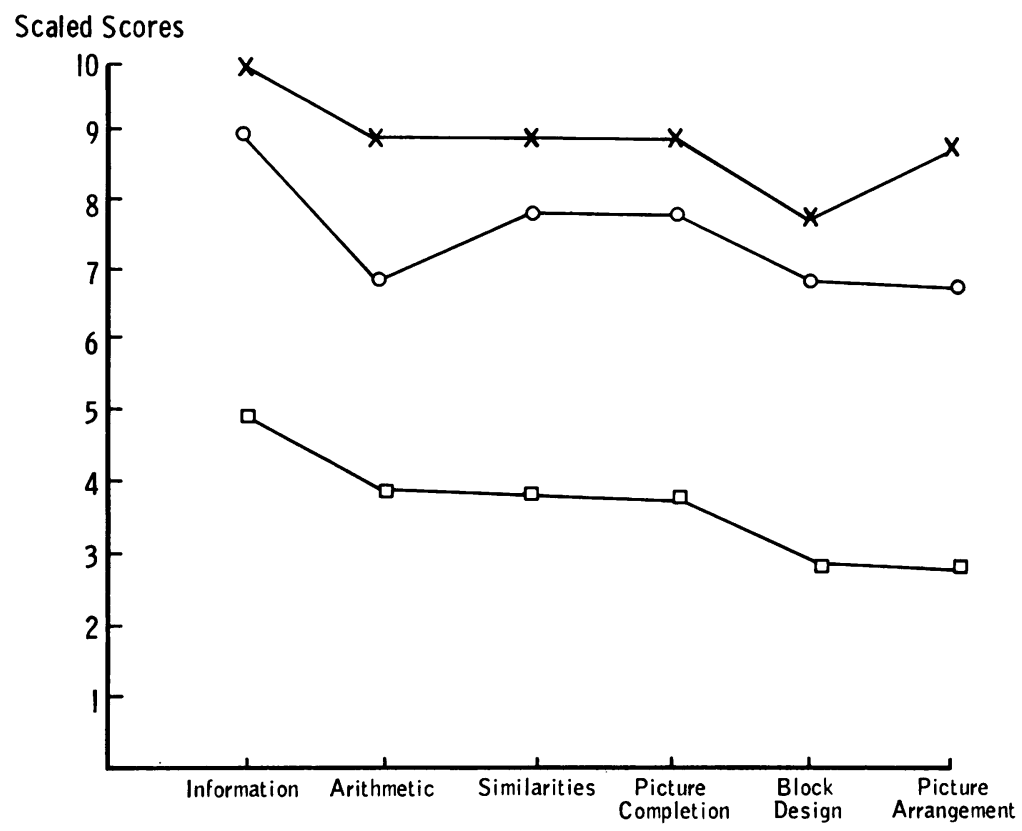

FIGURE Age corrected scaled scores for individual WAIS subtests. $\times-\times$ Vertebrobasilar insufficiency. $\bigcirc-\bigcirc$ Multi-infarct dementia. $\square-\square$ Alzheimer's disease.

categories. This analysis classified Block Design as the best individual discriminating variable. The addition of the Similarities subtests raw score increased the diagnostic accuracy of the discriminant function analysis. The data set out in Table 7 suggest that misclassification of patients was due to their individual performances on these subtests. These results indicate that among the three groups (VBI, MID, and AD), the diagnosis was more easily made when tasks measuring visual motor coordination and abstract reasoning were included in the analysis. If limited neuropsychological evaluation is available, these two subtests are optimum for diagnostic classification. Future studies for assessing the natural history of the course of the disease and studies assessing the effects of various approaches to treatment should include measures of abstract reasoning and visual-motor coordination in their analysis.

There are important implications resulting from the present findings. First of all, similar patterns of cognitive and intellectual impairment were found in all three diagnostic groups. These patterns, however, were not task specific, differing only in the degree of impairment. It was found that the three diagnostic groups differed
TABLE 7

AGE CORRECTED SCALED SCORES FOR BLOCK DESIGN AND SIMILARITIES FOR THOSE PATIENTS MISCLASSIFIED BY THE DISCRIMINANT FUNCTION ANALYSIS

\begin{tabular}{lrrl}
\hline Neurologist's diagnosis & $\begin{array}{l}\text { Block } \\
\text { design }\end{array}$ & Similarities & $\begin{array}{l}\text { Discriminant } \\
\text { classification }\end{array}$ \\
\hline 1. Multi-infarct dementia & 8 & 7 & VBI \\
2. Multi-infarct dementia & 0 & 0 & Alzheimer's \\
3. Multi-infarct dementia & 0 & 0 & Alzheimer's \\
4. Multi-infarct dementia & 11 & 12 & VBI \\
5. Multi-infarct dementia & 11 & 14 & VBI \\
6. Multi-infarct dementia & 0 & 0 & Alzheimer's \\
7. Multi-infarct dementia & 7 & 6 & VBI \\
8. Alzheimer's disease & 0 & 6 & Multi-infarct \\
9. Vertebrobasilar insufficiency & 10 & 13 & Multi-infarct \\
10. Vertebrobasilar insufficiency & 6 & 4 & Multi-infarct \\
11. Vertebrobasilar insufficiency & 10 & 11 & Multi-infarct \\
& & & \\
\hline
\end{tabular}

mostly in their performance on tasks requiring visual-motor coordination and abstract reasoning. These tasks were also found by Crookes (1974) to be the best indices of early dementia utilizing the WAIS. If these findings are consistently replicated, it might be possible to develop an 'early warning system' in order to detect a dementing process at its onset and possibly institute remedial or preventive measures. 
Of particular interest is the overall cognitive performance of the VBI group. It was only recently that Djibladse (1973) made the important observation for the first time that cases of long standing vertebrobasilar insufficiency develop a characteristic dementia which should be considered part of the natural history of the disease. Transient global amnesia and amnesic stroke may also be later complications of VBI, because the terminal branches of the vertebrobasilar system supply those parts of the diencephalon, para-amygdaloid areas as well as other parts of the temporal and occipital lobes concerned with important intellectual functions, particularly recent memory and visual-spatial functioning (Benson et al., 1973; Mathew and Meyer, 1974). The present results confirm that patients with VBI perform slightly below the average on most cognitive measures when their scores are corrected for age. However, their poorest performance was on Block Design, a task measuring visual-motor coordination, which was markedly reduced when compared with agematched norms. It is probable that with more detailed and specifically designed assessments of cognitive functioning even slighter degrees of deterioration earlier in the process can be identified. Early recognition of dementia in the VBI patient is important because there are now several reports that medical treatment may improve cognitive performance if instituted early in the disease (Rivera et al., 1974, 1975).

Multiple infarcts can produce a condition in which dementia may be the dominant symptom. This condition has been correctly named by Hachinski et al. (1974) as multi-infarct dementia (MID). This was to escape from the pejorative and confusing term 'arteriosclerotic dementia' in explaining a gradual 'brain failure' due to a slowly progressive diffuse reduction in cerebral circulation. Such a concept is inaccurate and leads to erroneous classifications of $\mathrm{AD}$ as 'arteriosclerotic dementia' and vice versa, and possible treatments of the three conditions, AD, VBI, and MID, may eventually prove to be entirely different. The present findings indicating striking variability in the degree of cognitive deficit within the MID group support these views. There is no doubt that there is a direct relationship between the site, multiplicity, and severity of the infarcts and the degree of intellectual and cognitive impairment.

To us the most significant implication of this study is the relative efficacy of the quantitative neuropsychological approach to the differential diagnosis of patients with dementia. We are currently analysing further tests which include detailed assessment in memory, language, and perceptual-motor functions which appear to enhance further the discriminatory accuracy of neuropsychological tests. These tests are potentially useful for studying the natural history of the diseases that produce dementia, as well as for assessing the possible benefits of various therapeutic trials in this growing public health problem of our aging populations.

\section{REFERENCES}

Baer, P. E., and Gaitz, C. M. (1971) Survival of elderly psychiatric patients. In Prediction of Life Span, pp. 153166. Edited by E. Palmore and F. C. Jeffers. Heath: Lexington, Mass.

Benson, D. F., Marsden, C. D., and Meadows, J. C. (1973). The amnesic stroke. (Abstract.) Neurology (Minneap.), 23, 400.

Crookes, T. G. (1974). Indices of early dementia on WAIS. Psychological Reports, 34, 734.

Djibladse, D. N. (1973). Diagnosis of occlusive disease of the extracranial arteries from their initial clinical manifestations of cerebral circulatory insufficiency. In Cerebral Vascular Disease, pp. 22-24. 6th International Conference, Salzburg, 1972. Edited by J. S. Meyer, H. Lechner, M. Reivich, and O. Eichhorn. Thieme: Stuttgart.

Fisher, C. M. (1968). Dementia in cerebral vascular disease. In Cerebral Vascular Diseases, pp. 232-236, Edited by J. F. Toole, R. G. Siekert, and J. P. Whisnant. Grune and Stratton: New York.

Fisher, R. A. (1936). Statistical Methods for Research Workers, 6th edn. Oliver and Boyd: Edinburgh.

Hachinski, V. C., Lassen, N. A., and Marshall, J. (1974). Multi-infarct dementia. A cause of mental deterioration in the elderly. Lancet, 2, 207-210.

Horenstein, S. (1971). The clinical use of psychological testing in dementia. In Dementia, pp. 61-80. Edited by C. E. Wells. Davis: Philadelphia.

Kannel, W. B., Blaisdell, F. W., Gifford, R., Hass, W., McDowell, F., Meyer, J. S., Millikan, C. H., Rentz, L. E., and Seltser, R. (1971). Risk factors in stroke due to cerebral infarction. Stroke, 2, 423-428.

Karp, H. (1974). Dementias in adults. In Clinical Neurology, 3rd edn, vol. 2. Edited by A. B. Baker and L. H. Baker. Harper and Row: Hagerstown, Md.

Mathew, N. T., and Meyer, J. S. (1974) Pathogenesis and natural history of transient global amnesia. Stroke, 5, 303-311.

Mathew, N. T., Meyer, J. S., Hartmann, A., and Ott, E. O. (1975). Abnormal CSF-blood flow dynamics in normal pressure hydrocephalus. Archives of Neurology. (In press.) 
Rivera, V. M., Meyer, J. S., Baer, P. E., and Faibish, G. M. (1975). Vertebrobasilar insufficiency as a cause of dementia. Controlled therapeutic trial with betahistine-HCl. In Cerebral Vascular Disease. 7th International Conference, Salzburg, 1974. Edited by J. S. Meyer, H. Lechner, and M. Reivich. Thieme: Stuttgart. (In press.)
Rivera, V. M., Meyer, J. S., Baer, P. E., Faibish, G. M., Mathew, N. T., and Hartmann, A. (1974). Vertebrobasilar arterial insufficiency with dementia. Controlled trials of treatment with betahistine hydrochloride. Journal of the American Geriatrics Society, 22, 397-406.

Wechsler, D. (1958) The Measurement and Appraisal of Adult Intelligence, 4th edn. Williams and Wilkins: Baltimore. 